\title{
Kinetic Equations: A French History
}

Claude Bardos (Université Pierre et Marie Curie and CNRS, Paris, France and WPI Wien, Austria) and Norbert J. Mauser (Wolfgang Pauli Institute and Universität Wien, Austria)

\section{Introduction}

In this article, we aim to describe the evolution of the mathematical study of kinetic equations between 1970 and 2000, covering French activity in particular. Of course, the subject being broad, we are going to focus on certain aspects. Particular attention will be paid to the role of the "averaging lemmas" as a crucial tool in classical kinetic theory. In this context, we are also going to present the Wigner transform in quantum kinetic theory, as well as the link to classical physics, in the "semi-classical" limit $\hbar \rightarrow 0$.

Asymptotic analysis will be a central topic in the following. For this reason, we are going to trace the development from ("mesoscopic") kinetic equations, like the Boltzmann equation, to ("macroscopic") fluid equations, like the Euler or Navier-Stokes equations, when certain parameters (Strouhal and Knudsen numbers) tend to zero. In order to place things in their context, it seemed important to us to have a look back and recall the pioneers. From the decade of 2000 on, however, the subject in a way "exploded" so that many additional papers would be necessary to expose it adequately.

To situate kinetic equations in a broader perspective, it seems advisable to remark that it took humanity thousands of years to invent, with Leibniz in 1684 and Newton in 1713, differential equations, well suited for the description of movement, while the invention of partial derivatives, describing continuous media, then took only 30 years, for example with d'Alembert in 1747 and Euler in 1775. In this context, for rarefied media, the notion of a "particle density" with a speed $v$ at a point $x$ is due to Maxwell in 1866, who also proposed the speed distribution that today bears his name. The "kinetic" equations, dedicated to the evolution of that density, appear subsequently with Boltzmann in 1872 and Lorentz in 1905. The importance of Boltzmann's ideas may not have been recognised immediately but they have played an essential role in mathematics since the beginning of the 20th century, through Hilbert's $6^{\text {th }}$ problem, exposed by him at the International Congress of Mathematicians (ICM) in 1900 in Paris, as well as through several contributions made by Einstein.

However, it was not before 1970 that mathematicians, and in particular French mathematicians, developed a genuine interest in the subject. As an example, at the ICM in Nice in 1970 , there was only one single talk (by Guiraud) related to this problem. One may also quote, regarding that period, some articles by specialists in mechanics, like Choquet-Bruhat and Bancel in 1973 and, of course, Cabannes in 1962, who was subsequently going to play a major role.

The subject gained growing importance in our community and in particular in France, as proved by the Fields Medals for P.-L. Lions (1994) and for Villani (2010), as well as numerous other international prizes.
This is certainly, among other reasons, due to the fact that kinetic equations appear in a broad variety of sciences: astrophysics, spaceflight (especially regarding the re-entry of vehicles into the atmosphere), interaction between fluids and particles, nuclear physics, semi-conductor technology and biology (for modelling cell evolution in immunology and chemotaxis). In addition, research in this field requires both pure and applied mathematics, with results of a geometric nature or related to harmonic analysis, probability and numerical methods. This explains why the subject is part of the expansion and globalisation of mathematical research. Many Summer (or Winter) schools have been dedicated to it, leading to diverse international collaborations, for example the GdR SPARCH (led by Raviart) and, in particular, the European network HYKE (HYperbolic and Kinetic Equations, led by N. M.), which unified the communities of "kinetic equations" and "hyperbolic conservation laws". It is certainly impossible to describe, in detail, all the mentioned topics at once, therefore we, as two active witnesses of the evolution in France and Europe, chose to focus on the years 1970-2000, with the history of "averaging lemmas" being a central thread.

\section{The time of the physicists and prehistory}

The kinetic equations concern quantities $f(x, v, t)$, with $f \geq 0$ representing a density (in the sense of a probability) of particles situated at a point $x$ and time $t$, additionally depending on a kinetic variable $v$. In the initial examples, $v$ represents the speed of the particles, so $f$ is the density of particles at point $x$ with speed $v$ at time $t$. We call $\mathbb{R}_{x}^{d} \times \mathbb{R}_{v}^{d}$ "the phase space" in $d$ space dimensions ( $d=3$ or $d=2,1$ for systems with symmetry or confinement). The speed $v$ can be replaced by a "momentum" $\xi$, etc., or a length and a direction when the objects are polymers or biological cells.

The kinetic equations contain by nature a "free transport" term (= "advection term"): $\partial_{t} f+v \cdot \nabla_{x} f$, which represents the evolution of the particle density in the absence of exterior forces. In this simple case, for an initial datum $f_{0}=f(t=0)$, the solution of the equation

$$
\partial_{t} f+v \cdot \nabla_{x} f=0
$$

will be

$$
f(x, v, t)=f_{0}(x-v t, v) .
$$

In the presence of an external force $F$ (gravity, electric force, etc.), we have to add to this free transport a term that corresponds to Newton's second law $\frac{d}{d t} v=F$ (mass $m=1$ ). If this force comes from a potential, i.e., $F=-\nabla_{x} V$, we have a so-called Liouville equation:

$$
\partial_{t} f+v \cdot \nabla_{x} f-\nabla_{x} V \cdot \nabla_{v} f=0, f(x, v, 0)=f_{0}(x, v),
$$

which, with the Poisson bracket

$$
\{H, f\}=\nabla_{v} H \cdot \nabla_{x} f-\nabla_{x} H \cdot \nabla_{v} f
$$


of the Hamiltonian $H(x, v)$, i.e., the energy $\frac{|v|^{2}}{2}+V(x)$, and the function $f$, can also be expressed in its symplectic form: $\partial_{t} f+\{H, f\}=0$. If the force $F$ depends on the solution $f$, Equation (1) becomes nonlinear, which is the case of the Vlasov equation (see Section 6).

With the kinetic equations describing intermediate regimes between the dynamics of particles and macroscopic observables, certain parameters (measuring, for instance, the rarefaction of the environment or the time scale) appear naturally. In particular, the Strouhal number, St, gives the timescales, while the Knudsen number, Kn, describes the density of the medium (also called the "mean free path"). Finally, a mathematical analysis leads to the introduction of a small reference parameter, denoted by $\epsilon$, to be compared to the other parameters.

We consider three equations in order to illustrate this analysis. We consider the Boltzmann equation

$$
\text { St } \partial_{t} f+v \cdot \nabla_{x} f=\frac{1}{\epsilon} C(f, f),
$$

the Lorentz equation

$$
\begin{aligned}
\epsilon \partial_{t} f & +v \cdot \nabla_{x} f \\
& =-\frac{1}{\epsilon} \int_{\mathbb{R}^{d}} k(x, v, w)(f(t, x, v)-f(t, x, w)) d \mu(w)
\end{aligned}
$$

and, with $S^{2}$ being the unit sphere of $\mathbb{R}^{3}$, an equation for a simplified model of the transport:

$$
\epsilon \partial_{t} f+\omega \cdot \nabla_{x} f=-\frac{1}{\epsilon}\left(f-\int_{S^{2}} f\left(x, \omega^{\prime}, t\right) d \omega^{\prime}\right) .
$$

In the Boltzmann equation (3), $C(f, f)$ represents the changes in particle speed due to elastic binary collisions, i.e., conserving the mass, linear momentum and the energy of the two colliding particles. In the scope of this article, we omit details of its structure; it is sufficient to keep in mind that one has:

$$
\begin{aligned}
& \int_{\mathbb{R}_{v}^{d}} C(f, f) d v=0, \quad \int_{\mathbb{R}_{v}^{d}} C(f, f) v d v=0, \\
& \int_{\mathbb{R}_{v}^{d}} C(f, f) \frac{|v|^{2}}{2} d v=0,
\end{aligned}
$$

as well as the decrease of the "entropy production", which vanishes for a Maxwellian distribution. It follows that

$$
\int_{\mathbb{R}_{v}^{d}} C(f, f) \log f d v \leq 0
$$

and, moreover, if

$$
\int_{\mathbb{R}_{v}^{d}} C(f, f) \log f d v=0
$$

then

$$
f(x, v, t)=M_{\rho, u, \theta}(x, v, t)=\frac{\rho}{(2 \pi \theta)^{\frac{d}{2}}} e^{-\frac{|v-u|^{2}}{2 \theta}},
$$

where $\rho(x, t), u(x, t)$ and $\theta(x, t)$ are the macroscopic densities characterising the Maxwellian distribution.

The relation (7) yields, in particular, the decrease of entropy

$$
\frac{d}{d t} \int_{\mathbb{R}_{x}^{d} \times \mathbb{R}_{v}^{d}} f(x, v, t) \log f(x, v, t) d x d v \leq 0,
$$

which is the famous $H$-theorem, a subject of controversies in Boltzmann's time as it seemed to be in apparent contradiction with Poincaré's recurrence principle. One can say that, at present, this paradox has been solved. The Lorentz equation (4) describes a situation in which the dominant process is the interaction of particles with an environment while the interaction between the particles is neglected. This explains why the equation is linear. It was introduced by Lorentz in 1905 for the evolution of electrons between atoms. Subsequently, it has played an essential role in the study of the interaction between neutrons and atomic nuclei. Here, $k(x, v, w)$ represents a positive and symmetric nucleus while $d \mu(w)$ is a probability on $\mathbb{R}_{v}^{d}$. The parameter $\epsilon$ is introduced to validate macroscopic approximations.

The "simplified transport model" (5) is an adaptation of the Lorentz equation (4) and corresponds to a measure $d \mu$ only supported on the unit sphere, so the absolute value of the speed is not affected by the interaction.

Concerning the Boltzmann equation, one obtains from the entropy balance that

$$
\begin{aligned}
\frac{d}{d t} & \iint_{\mathbb{R}^{d} \times \mathbb{R}^{d}} f_{\epsilon}(x, v, t) \log f_{\epsilon}(x, v, t) d x d v \\
& +\frac{1}{\epsilon} \frac{d}{d t} \iint_{\mathbb{R}^{d} \times \mathbb{R}^{d}} f_{\epsilon}(x, v, t) C\left(f_{\epsilon}, f_{\epsilon}\right) \log f_{\epsilon}(x, v, t) d x d v=0 .
\end{aligned}
$$

From Equation (8), one also infers that, for $\epsilon \rightarrow 0$, any adherence value of the family $f_{\epsilon}$ is a local Maxwellian, i.e., a Gaussian $M$ given by (8). By inserting this expression into the Boltzmann equation and by using Equation (6) of momentum conservation, one additionally deduces that the macroscopic parameters are the solution of the Euler equations of compressible fluids:

$$
\begin{aligned}
& \text { St } \partial_{t} \rho+\nabla_{x} \cdot(\rho u)=0 \\
& \text { St } \partial_{t}(\rho u)+\nabla_{x} \cdot(\rho u \otimes u+\rho \theta)=0 \\
& \text { St } \partial_{t}\left(\rho \frac{|u|^{2}}{2}+\frac{d}{2} \rho \theta\right)+\nabla_{x} \cdot\left(u\left(\rho \frac{|u|^{2}}{2}+\frac{d+2}{2} \rho \theta\right)\right)=0 .
\end{aligned}
$$

This derivation (in a more modern form, of course) motivated Hilbert when he announced his $6^{\text {th }}$ problem at the ICM in Paris in 1900:

Boltzmann's work on the principles of mechanics suggests the problem of developing mathematically the limiting processes, there merely indicated, which lead from the atomistic view to the laws of motion of continua.

On the other hand, we observe that Equations (9) expresses neither the viscosity nor the thermal diffusivity of the fluid (fundamental quantities in fluid mechanics). To solve the problem, Hilbert suggests in 1916 to express these quantities in the second term in $\epsilon$ of a formal development of the function $f_{\epsilon}$. Independently, Chapman in 1916 and Enskog in 1917 established a more direct connection between the Boltzmann equation and the macroscopic equations. Rather than considering the first two terms of the development in $\epsilon$, they introduce a local Maxwellian (8), which depends on the parameter

$$
M_{\epsilon}(x, v, t)=\frac{\rho_{\epsilon}}{\left(2 \pi \theta_{\epsilon}\right)^{\frac{d}{2}}} e^{-\frac{\left|v-u_{\epsilon}\right|^{2}}{2 \theta_{\epsilon}}},
$$

and they prove that, for $M_{\epsilon}$ to be a solution of the Boltzmann equation up to an $\epsilon^{2}$-order term, it is necessary and sufficient that $\rho_{\epsilon}, u_{\epsilon}, \theta_{\epsilon}$ are solutions of the "macroscopic" Navier- 
Stokes equations

$$
\begin{aligned}
& \operatorname{St} \partial_{t} \rho_{\epsilon}+\nabla_{x}\left(\rho_{\epsilon} u_{\epsilon}\right)=0, \\
& \begin{aligned}
\operatorname{St} \partial_{t}\left(\rho_{\epsilon} u_{\epsilon}\right) & +\nabla_{x}\left(\rho_{\epsilon} u_{\epsilon} \otimes u_{\epsilon}+\rho_{\epsilon} \theta_{\epsilon}\right) \\
& =\epsilon \nabla_{x} \cdot\left\{v\left(\theta_{\epsilon}\right)\left(\sigma\left(u_{\epsilon}\right)-\frac{2}{d} \nabla_{x} \cdot \sigma\left(u_{\epsilon}\right) \mathrm{I}\right)\right\},
\end{aligned} \\
& \begin{aligned}
\operatorname{St} \partial_{t}\left(\rho_{\epsilon} \frac{\left|u_{\epsilon}\right|^{2}}{2}\right. & \left.+\frac{d}{2} \rho_{\epsilon} \theta_{\epsilon}\right)+\nabla_{x}\left(u_{\epsilon}\left(\rho \frac{\left|u_{\epsilon}\right|^{2}}{2}+\frac{d+2}{2} \rho_{\epsilon} \theta_{\epsilon}\right)\right) \\
& =\frac{\epsilon v\left(\theta_{\epsilon}\right)}{2} \sigma\left(u_{\epsilon}\right): \sigma\left(u_{\epsilon}\right)+\epsilon \nabla_{x} \cdot\left[\kappa\left(\theta_{\epsilon}\right) \nabla_{x} \theta_{\epsilon}\right],
\end{aligned}
\end{aligned}
$$

with

$$
\sigma\left(u_{\epsilon}\right)=\frac{\nabla u_{\epsilon}+\nabla^{t} u_{\epsilon}}{2}, \quad \sigma\left(u_{\epsilon}\right): \sigma\left(u_{\epsilon}\right)=\operatorname{Trace} \sigma\left(u_{\epsilon}\right) \otimes \sigma\left(u_{\epsilon}\right) .
$$

Thus, the viscosity $\epsilon \mathcal{V}\left(\theta_{\epsilon}\right)$ and the thermal diffusivity $\epsilon \kappa\left(\theta_{\epsilon}\right)$, two macroscopic quantities that in Boltzmann's or Maxwell's time were already experimentally measurable, are, in the above derivations, quantities that are proportional to the Knudsen number. They depend on the macroscopic temperature according to the power laws, which can be intuited (and even computed) from the interaction between the molecules. The accordance between the experimentally obtained measurements and the formulas deduced from the above calculation allowed the confirmation of the hypotheses on the dynamics of those molecules.

Some of the above calculations are very formal: as all these equations are nonlinear, studying them rigorously was only possible with the modern tools of functional analysis. In particular, the Euler equations support singular solutions (with discontinuities) and under natural conditions of compression, regular solutions become singular at finite time. They are good examples of notions of derivatives being used in the sense of distributions and, hence, the solutions are weak. We observe that the relation of conservation of the macroscopic entropy can be derived from these equations:

$$
\partial_{t}\left(\rho \log \frac{\rho^{\frac{2}{3}}}{\theta}\right)+\nabla_{x} \cdot\left(\rho u \log \frac{\rho^{\frac{2}{3}}}{\theta}\right)=0 .
$$

However, this type of computation is no longer valid in the presence of discontinuities. One can also demonstrate that, if the weak solutions of the compressible Euler equations are weak limits, in a convenient sense, of solutions to NavierStokes equations or moments of solutions to the Boltzmann equation, they only satisfy Relation (12) in the sense of an inequality (it turns out that the left side is negative or zero). In one space dimension, this constraint ensures the uniqueness and stability of the corresponding solutions. In more than one dimension, however, the recent works of Chiodaroli, De Lellis and Kreml [8], following up on the results obtained by De Lellis and Szkelyhidi, have proven a total instability (even an infinite number of entropic solutions) for the Euler equations.

Extracting what can be rigorously proven (considering the available tools) is the aim of the research activity of the years 1970-2000, which mainly involves incompressible solutions of Navier-Stokes and fluctuations of renormalisable solutions (in the sense of DiPerna-Lions) of the Boltzmann equation. This is a central object of this article (see Section 4).

\section{The CEA and the transport equation}

As mentioned above, after Hilbert and during the interwar period, we hardly find any research on this subject led by French mathematicians. They seemed to have forgotten, for example, Poincare as well as Hilbert's $6^{\text {th }}$ problem. On the other hand, there is at least a physicist, Jacques Yvon, who had already formulated problems in a new language, the interest of which would be understood only many years later by the mathematicians. In 1936 (see [23]), he introduced, in order to examine a gas of $N$ molecules, in addition to the densities of $n \leq N$ particles $f_{n}^{N}\left(x_{1}, x_{2}, \ldots, x_{n}, v_{1}, v_{2}, \ldots, v_{n}\right)$, the joint probability to have the first particle at position $x_{1}$ with speed $v_{1}$, the second at position $x_{2}$ with speed $v_{2}$, etc., and he made explicit the relations between those densities. Every density $f_{n}^{N}$ is, for $n<N$, a solution of an equation evolving in its second term the density $f_{n+1}^{N}$. And, of course, $f_{N}^{N}$ coincides with the solution of the Liouville equation determined by the initial system. He demonstrated that the solutions of the Boltzmann equation produce, by factorisation, approximate solutions of this system of equations. He also invented a hierarchy of equations that would be rediscovered 11 years later by Kirkwood, Born and Green, as well as by Bogoliubov, and would therefore be named BBGKY hierarchy. Besides its very concrete interpretation in physics, this hierarchy was going to play an important role in mathematical proofs, starting with the works of Grad in 1949 and Lanford in 1974 on the rigorous derivation of the Boltzmann equation.

The "Commissariat à l'Energie Atomique" (CEA) was founded in 1945 and Yvon entered it in 1946, first as a collaborator, secondly as an "external member" in 1949 and finally becoming high-commissioner from 1970 to 1975.

On the transport equation of neutrons, he wrote: "I soon understood (1946) that Boltzmann's integro-differential equation, slightly modified, would serve as an arsenal for the new mathematical physicists." As mentioned above, it is, in fact, an application of the Lorentz equation to interactions (absorption and re-emission) of neutrons with surrounding atoms. This equation, which of course does not have an explicit solution, forms part of the challenges that the CEA would face with the help of computers and more mathematics. It is the programme of Amouyal and Horowitz, defined under the influence of Yvon and within this framework, where Dautray was to become, in 1955, part of the CEA's mathematical physics group. That's where J.-L. Lions met him as an external collaborator introduced by Lattes.

Thanks to the participation and financial support of the CEA and industry partners like EDF and Dassault, and with the collaboration of some former students of J.-L. Lions, this group played a major role in the expansion of applied mathematics in the 1950s, where kinetic equations were important, initially through the organisation of Summer schools like the CEA-EDF-INRIA at Bréau Castle (following the example of the physics schools of Houches and, later on, Cargèse), which are very popular in our community.

His position as a university professor allowed J.-L. Lions to invite some of the leading mathematicians in the domain of kinetic equations to extended stays in France: Nishida to Orsay in 1974, Ukai to Orsay and Paris 13 in 1977, Nico- 
laenko to Orsay in 1977 and Papanicolaou to the INRIA and the Summer school at Bréau Castle in 1978.

Finally, there is an important publication that should be explicitly highlighted. Dautray took the initiative to coordinate, together with J.-L. Lions, the elaboration of a treatise in applied mathematics "Analyse Mathématique et Calcul Numérique pour les Sciences et la Technologie". The first of the 9 volumes was published in 1984 by Masson, Paris, and there were subsequently various versions and translations, in particular one published by Springer in English.

The initiative was inspired by the work of the Bourbaki group on one hand and Courant-Hilbert, a seminal book of mathematical physics, on the other. J.-L. Lions and Dautray followed the structure of this reference book regarding its subjects, its volumes and chapters but they delegated, even more than Courant-Hilbert (where the contributions of Friedrichs, John, Lax, Nirenberg and others are mentioned), the writing of chapters and whole volumes of the work to younger researchers, quoting them entirely. Following Bourbaki's tradition in optimising editing, Dautray gathered the writers and made the different contributions circulate for reciprocal reviews.

At the time, he arranged convenient conditions for such an initiative. He had ensured the collaboration of various CEA researchers, among them Sentis and Kavenoky. He had Cessenat, a CEA engineer, working full-time on the project and could count on permanent consultants such as P.-L. Lions and Perthame. He had also ensured the collaboration of scientists doing their military service (which was still mandatory at the time). Among such scientists doing their military service were, in 1985, some of our (then juvenile) colleagues like Julia and Golse.

\section{Diffusion approximation}

Dautray and J.-L. Lions entrusted the redaction of a volume on the transport equation to C. B. In particular, Dautray insisted on the rigorous formulation of the diffusion approximation.

The evolution of neutrons in the presence of reactive nuclei (uranium or plutonium) is described by a kinetic equation of Lorentz type (4). However, as with every kinetic equation, it depends on the $2 d$ variables of the phase space and on the time variable. A direct calculation is thus not possible. Also in the 1940s-60s, Metropolis and Ulam at Los Alamos, Khasminski in Russia and Benoist at the CEA used, in order to calculate the "macroscopic" density of particles

$$
\rho(x, t)=\int_{\mathbb{R}^{d}} f(x, v, t) d v
$$

at point $x$ and time $t$, an approximation called diffusion approximation, which is defined by the solution of the equation:

$$
\partial_{t} \rho-\kappa \Delta \rho=c \rho .
$$

This approach (valid near the critical regime) was based on a scale estimate together with a probabilistic interpretation. This is, by the way, the procedure on which is based the socalled Monte Carlo method for calculating integrals and solutions of equations with partial derivatives. While the method was well explained from the angle of physics in the book by Weinberg and Wigner in 1958 [22], one was far from having a precise mathematical formulation allowing its justification.
In 1974, Larsen and Keller were inspired by the role of the Knudsen and the Strouhal number in the Boltzmann equation to produce a direct proof based on functional analysis. Following Dautray's suggestion, this proof was reproduced in the book. For better understanding, we also reproduce it here, in the framework of the following simplified model

$$
\partial_{t} f_{\epsilon}+\frac{1}{\epsilon} \omega \cdot \nabla_{x} f_{\epsilon}+\frac{1}{\epsilon^{2}}\left(f_{\epsilon}-\frac{1}{4 \pi} \int_{\mathbb{S}^{2}} f_{\epsilon}\left(x, \omega^{\prime}\right) d \omega^{\prime}\right)=c(x) f_{\epsilon},
$$

where $\omega \in \mathbb{S}^{2}, x \in \Omega \subset \mathbb{R}^{3}$ and $x \mapsto c(x)$ is a function depending only on $x$, positive or negative. Of course, it is convenient to add conditions on the limits. In neutron physics, the standard boundary condition is the absorbing condition (any particle that leaves the environment is absorbed by the surroundings and thus does not return). By introducing the external normal $\vec{n}$ to the boundary $\partial \Omega$, this can be expressed by the following:

$$
\forall(x, \omega) \in \partial \Omega \times \mathbb{S}^{2}, \quad \omega \cdot \vec{n}(x)<0 \Rightarrow f_{\epsilon}(x, \omega, t)=0 .
$$

Applying the Green formula, multiplication by $f_{\epsilon}$ and integration on $\partial \Omega \times \mathbb{S}^{2}$ leads to the inequality

$$
\begin{aligned}
& \frac{d}{d t} \frac{1}{2} \int_{\Omega \times \mathbb{S}^{2}}\left|f_{\epsilon}(x, \omega, t)\right|^{2} d \omega d x \\
& +\frac{1}{\epsilon^{2}} \int_{\Omega} d x \int_{\mathbb{S}^{2}}\left(f_{\epsilon}-\frac{1}{4 \pi} \int_{\mathbb{S}^{2}} f_{\epsilon}(x, \omega) d \omega\right)^{2} d \omega \\
& \quad \leq \int_{\Omega \times \mathbb{S}^{2}} c(x)\left|f_{\epsilon}(x, \omega, t)\right|^{2} d \omega d x .
\end{aligned}
$$

It turns out that the solutions are, for $0 \leq t \leq T$, uniformly bounded with respect to $\epsilon$ in $L^{2}\left(\Omega \times \mathbb{S}^{2}\right)$ and that, for $\epsilon \rightarrow 0$, any value of adherence of the sequence $f_{\epsilon}$ in $L^{2}$ is a function $\rho(x, t)$ that is independent of $\omega$, as will be the case for other weak limits $\overline{f_{\epsilon}}$. The integration of the relation (15) with respect to $\omega$ yields the conservation law:

$$
\begin{aligned}
\partial_{t} \frac{1}{4 \pi} \int_{\mathbb{S}^{2}} f_{\epsilon}(x, \omega) d \omega+\nabla_{x} & \cdot\left(\frac{1}{4 \pi \epsilon} \int_{\mathbb{S}^{2}} \omega f_{\epsilon}(x, \omega) d \omega\right) \\
& =c(x) \frac{1}{4 \pi} \int_{\mathbb{S}^{2}} f_{\epsilon}(x, \omega) d \omega .
\end{aligned}
$$

With $\int_{\mathbb{S}^{2}} \omega f_{\epsilon}(x, \omega) d \omega$ converging weakly to 0 , it is convenient (as has already been mentioned) to raise the indetermination in the second term of the first member of (18). To do this, we multiply the equation (15) a second time by $\epsilon \omega$ and obtain, after integration:

$$
\begin{aligned}
& \frac{1}{4 \pi \epsilon} \int_{\mathbb{S}^{2}} \omega f_{\epsilon}(x, \omega) d \omega \\
& =-\nabla_{x} \frac{1}{4 \pi} \int_{\mathbb{S}^{2}} \omega \otimes \omega f_{\epsilon}(x, \omega, t) d \omega \\
& \quad+\epsilon \frac{1}{4 \pi} \int_{\mathbb{S}^{2}} \omega\left(c(x) f_{\epsilon}(x, \omega, t)\right. \\
& \quad-\epsilon \partial_{t} f_{\epsilon}(x, \omega, t) d \omega \\
& \rightarrow \nabla_{x} \frac{1}{4 \pi} \int_{\mathbb{S}^{2}} \omega \otimes \omega d \omega: \nabla_{x} \overline{f(x, t)}=-\frac{1}{3} \nabla_{x} \rho(x, t) .
\end{aligned}
$$

By putting this into equation (18) and taking the limit (it is a linear problem and at this point the nature of the convergence does not need to be given), we obtain:

$$
\partial_{t} \rho(x, t)-\frac{1}{3} \Delta \rho(x, t)=c(x) \rho(x, t) .
$$


Now, the problem with the boundary condition on $\partial \Omega$ has to be solved. Taking (16) into account, the Dirichlet condition

$$
x \in \partial \Omega \Rightarrow \rho(x, t)=0
$$

seems to be the most natural. However, in the 1950s, physicists (see page 198 of [22]) observed that the approximation was much better if one replaces the Dirichlet condition by a condition of the Robin type:

$$
x \in \partial \Omega \Rightarrow \rho(x, t)+\lambda \partial_{\vec{n}} \rho(x, t)=0 .
$$

The term $\lambda$ with the dimension of length is called extrapolation length. Its evaluation is inspired by the observation of stellar radiation.

While writing the Dautray-Lions volume, with the scales that Larsen and Keller proposed, the direct (quantitative) demonstration for the calculation of this $\lambda$ was found, by analysing the transport problem in a half-space named Milne space (after the astrophysician).

It turns out that this problem appears in an analogous manner in the relation between the Boltzmann equation and the compressible Navier-Stokes equation (11). The adaptation of the results obtained in Milne space to the Boltzmann equation was considered in 1986 in an article by Bardos, Caflish and Nicolaenko [1]. Also, this type of research was applied in the space shuttle project HERMES, planned in 1975 but then abandoned in 1992. Being a European project, it required regular collaborations between industry and university researchers, amongst them, in particular, (together with the French scientists) Neunzert from Kaiserlautern and Cercignani from the Politecnico de Milan (who had worked continuously and very successfully on the Boltzmann equation since 1962).

This collaboration also continued beyond the European borders, with Desphande (Indian Institute of Science, Bangalore), for example, and especially with Sone and his group at the Laboratory of Aeronautical Engineering in Kyoto.

\section{Approximation of the critical size}

In a volume dedicated to the transport equation and edited by the CEA, it was natural to evoke the critical size problem, which, in the kinetic regime, is expressed by the principal eigenvalue of the (unbounded) operator, defined on $L^{2}\left(\Omega \times \mathbb{R}_{v}^{d}\right)$ by

$$
T(f)=f \mapsto-v \cdot \nabla_{x} f+\mathcal{L} f,
$$

with a convenient boundary condition (for example, the absorbing one), while $\mathcal{L}$ is a linear operator that acts on the variables $v$ and represents the effects of the environment on the particles (absorption and re-emission). The Lorentz equations (4) and their simplification (5) give the most significant prototypes. The spectral analysis of the operator $f \mapsto T(f)$ is not simple because it is neither selfadjoint nor anti-selfadjoint and its spectrum may contain, at the same time, a continuous spectrum and eigenvalues with finite multiplicity.

Nevertheless, mathematicians like Albertoni- Montagnini in 1966 and Ghidouche-Point-Ukai in 1976 have demonstrated the existence of a real and simple principal eigenvalue. It is thus natural to expect that the eigenvalue obtained by the diffusion approximation would deliver a "good approximation" of the principal eigenvalue $\Lambda_{\epsilon}$ of the transport operator and therefore contribute to determining the critical character of the material.

In the case of the simplified model (5), using the scale change by Larsen and Keller, we are led to consider the pair $\left(\Lambda_{\epsilon}, f_{\epsilon}(x, v) \geq 0\right)$ as a solution of the equation (with absorbing boundary conditions):

$$
\begin{aligned}
& -\frac{1}{\epsilon} \omega \cdot \nabla_{x} f_{\epsilon}-\frac{1}{\epsilon^{2}}\left(f_{\epsilon}-\frac{1}{4 \pi} \int_{\mathbb{S}^{2}} f_{\epsilon}\left(x, \omega^{\prime}\right) d \omega^{\prime}\right)+c(x) f_{\epsilon}=\Lambda_{\epsilon} f_{\epsilon}, \\
& f_{\epsilon}(x, v) \geq 0, \quad \int_{\Omega \times \mathbb{S}^{2}}\left|f_{\epsilon}(x, \omega)\right|^{2} d x d \omega=1 .
\end{aligned}
$$

In a paper [18], which was part of his "Thèse d'Etat" defended in 1981, Sentis proved that the pair $\left(\Lambda_{\epsilon}, f_{\epsilon}\right)$ converges to $(\Lambda, u)$, a solution of the diffusion equation with Dirichlet boundary conditions:

$$
\frac{1}{3} \Delta u+c(x) u=\Lambda u, \quad u=0 \text { on } \partial \Omega .
$$

The aim was also to demonstrate that, with the introduction of the extrapolation length $\lambda$ given by Milne's problem, the principal eigenvalue corresponding to the same operator with the Robin condition

$$
u_{\epsilon}+\epsilon \lambda \partial_{\vec{n}} u_{\epsilon}=0 \quad \text { on } \quad \partial \Omega
$$

should give an approximation of higher order.

These results were then included in the last chapter of Dautray-Lions' book on transport. But, meanwhile, toward the end of 1984, Cessenat, entrusted by Dautray with the final proofreading of the different contributions, had discovered a "gap" in the proof. In the proof of the main result [18], it was not really established that $\int_{\mathbb{S}^{2}} f_{\epsilon}(x, \omega) d \omega$ converges to a nonzero function. He asked Sentis to solve the problem urgently (as the proofs needed to be sent to the editor). Sentis asked Golse, by that time working with him in the CEA as part of his military duties, and Perthame, also by that time a consultant at the CEA and sharing his office, to help him correct the proof.

It is clear that if $f \in L^{2}\left(\Omega \times \mathbb{S}^{2}\right)$ and $\omega \cdot \nabla_{x} f \in L^{2}\left(\Omega \times \mathbb{S}^{2}\right)$ then the function $x \mapsto f(x, \omega)$ possesses a supplementary regularity in the direction $\omega$ but that does not help further. In fact, they proved in a note to the CRAS, published at the beginning of 1985, that if the functions $f$ are bounded in $L^{2}\left(\Omega \times \mathbb{S}^{2}\right)$ and are such that $\left\|\omega \cdot \nabla_{x} f\right\|_{L^{2}\left(\Omega \times \mathbb{S}^{2}\right)}$ are bounded then, for every function $\phi \in L^{\infty}\left(\mathbb{S}^{2}\right)$, the averages $\int_{\mathbb{S}^{2}} f(x, \omega) \phi(\omega) d \omega$ form a relatively compact set in $L^{2}(\Omega)$. That lemma allowed the correction of the proof of the announced result. Later on, Golse, Perthame and Sentis showed, together with P.-L. Lions, that these averages belong to the Sobolev space $H_{x}^{\frac{1}{2}}$, that is, one gains half a notch of regularity in $x$. More precisely, as an example, we have [12]:

Theorem 3.1. For all test functions $\phi \in L^{\infty}\left(\mathbb{R}_{v}^{d}\right)$ with compact support, there exists a constant $C(\phi)$ such that if $f \in L^{2}\left(\mathbb{R}_{t} \times\right.$ $\left.\mathbb{R}_{x}^{d} \times \mathbb{R}_{v}^{d}\right)$ and

$$
\epsilon \partial_{t} f+v \cdot \nabla_{x} f=h \in L^{2}\left(\mathbb{R}_{t} \times \mathbb{R}_{x}^{d} \times \mathbb{R}_{v}^{d}\right)
$$

then

$$
\left\|\int f(x, v, t) \phi(v) d v\right\|_{L^{2}\left(\mathbb{R}_{t}, H^{\frac{1}{2}}\left(\mathbb{R}_{x}^{d}\right)\right)} \leq C(\phi)\|h\|_{L^{2}\left(\mathbb{R}_{t} \times \mathbb{R}_{x}^{d} \times \mathbb{R}_{v}^{d}\right)} .
$$


Proof. We denote by $\hat{f}(\xi, v, \tau)$ the Fourier transform with respect to $x, t$ of the function $f(x, v, t)$ and similarly for the function $\hat{h}$. We would like to bound

$$
|\xi|\left|\int \hat{f}(\xi, v, \tau) \phi(v) d v\right|^{2}
$$

With this aim, we introduce the parameter $\alpha$ and decompose the integral into two parts respectively to the sign of $\mid \epsilon \tau+\xi$. $v \mid-\alpha$. We bound

$$
\left|\int 1_{|\epsilon \tau+\xi \cdot \xi| \leq \alpha} \hat{f}(\xi, v, \tau) \phi(v) d v\right|^{2}
$$

by

$$
\left(\int|\hat{f}(\xi, v, \tau)|^{2} d v\right)\left(\int 1_{|\epsilon \tau+\xi \cdot v| \leq \alpha}|\phi(v)|^{2} d v\right) .
$$

Next, we note that $|(\epsilon \tau+\xi \cdot v) \hat{f}(\xi, v, \tau)|=|\hat{h}(\xi, v, \tau)|$ to bound

$$
\left|\int 1_{|\epsilon \tau+\xi \cdot v|>\alpha} \hat{f}(\xi, v, \tau) \phi(v) d v\right|^{2}
$$

by

$$
\left(\int|\hat{h}(\xi, v, \tau)|^{2} d v\right)\left(\int \frac{1_{|\epsilon \tau+\xi \cdot v|>\alpha}}{|\epsilon \tau+\xi \cdot v|^{2}}|\phi(v)|^{2} d v\right) .
$$

Both integrals depending on $\phi$ can directly be estimated. We conclude the proof with an optimal choice of $\alpha$.

Dautray later discovered that Theorem 3.1 had a forerunner. In 1984, Agoshkov established a result of that type. He used it for trace theorems that were very useful in numerical analysis. The broader significance of his formulation, however, he seemed to overlook.

\section{$4 \quad$ Application of averaging lemmas}

One of the challenges of the 1980s was the proof of the existence of solutions (possibly in a weak sense) for the Boltzmann equation for any natural initial datum, using only mass and energy conservation:

$$
\begin{aligned}
& \int_{\mathbb{R}_{x}^{d} \times \mathbb{R}_{v}^{d}}\left(1+\frac{|v|^{2}}{2}\right) f(x, v, t) d x d v \\
&=\int_{\mathbb{R}_{x}^{d} \times \mathbb{R}_{v}^{d}}\left(1+\frac{|v|^{2}}{2}\right) f(x, v, 0) d x d v,
\end{aligned}
$$

as well as the decrease of entropy:

$$
\begin{aligned}
& \int_{\mathbb{R}_{x}^{d} \times \mathbb{R}_{v}^{d}} f(x, v, t) \log f(x, v, t) d x d v \\
& \leq \int_{\mathbb{R}_{x}^{d} \times \mathbb{R}_{v}^{d}} f(x, v, 0) \log f(x, 0) d x d v,
\end{aligned}
$$

leading to the idea of adapting Theorem 3.1 to other functions spaces. So, in 1988, Golse, P.-L. Lions, Perthame and Sentis [12] demonstrated by interpolation that the relations

$$
f(x, v) \text { and } v \cdot \nabla_{x} f \in L^{p}\left(\mathbb{R}_{x}^{d} \times \mathbb{R}_{v}^{d}\right)
$$

yield, for $1<p<\infty$ (with $\phi \in L^{\infty}\left(\mathbb{R}_{v}^{3}\right)$ of compact support) and for $0<s<\inf (1 / p, 1-1 / p)$, the estimate

$$
\left\|\int_{\mathbb{R}_{v}^{d}} f(x, v) \phi(v) d v\right\|_{W^{s, p}} \leq C(\phi)\|f\|_{L^{p}\left(\mathbb{R}_{x}^{d} \times \mathbb{R}_{v}^{d}\right)}^{1-s}\left\|v \cdot \nabla_{x} f\right\|_{L^{p}\left(\mathbb{R}_{x}^{d} \times \mathbb{R}_{v}^{d}\right)}^{s} .
$$

Using a basic example, they observed that this estimate does not extend to the case $p=1$. In an attempt to overcome that obstacle, DiPerna and P.-L. Lions used, together with the averaging lemma, the a priori estimates of energy and especially entropy. This is how they came to prove the existence of solutions for the Boltzmann equation (in a relatively weak sense) called renormalised but global in time and depending only on the natural properties of the initial data (see [6]).

The analogy between this proof and the one given by Leray for the Navier-Stokes equations is striking, both for the results and the methods. It therefore becomes intuitive to have, at the macroscopic limit of the Boltzmann equation, "turbulent" Leray solutions of incompressible Navier-Stokes equations $(\nabla \cdot u=0)$ with strictly positive viscosity.

In 1991, Bayly, Levermore and Passot [3] observed that, if we introduce into the equations (11) a speed as well as fluctuations of density and temperature of order $\epsilon:(u, \rho, \theta)=$ $(\epsilon \tilde{u}, 1+\epsilon \tilde{\rho}, 1+\epsilon \tilde{\theta})$, we obtain formally, within the limit $\epsilon \rightarrow 0$, the incompressible Navier-Stokes equations with, in particular, strictly positive viscosity $v^{*}$ and thermal diffusiveness $\kappa^{*}$.

In order to link the solutions found by DiPerna-Lions to those given by Leray, it is convenient to consider functions

$$
f_{\epsilon}=\frac{1}{(2 \pi)^{\frac{d}{2}}} e^{-\frac{||^{2}}{2}}\left(1+\epsilon g_{\epsilon}(x, v, t)\right),
$$

solutions of re-scaled Boltzmann equations:

$$
\epsilon \partial_{t} f_{\epsilon}+v \cdot \nabla_{x} f_{\epsilon}=\frac{1}{\epsilon} C\left(f_{\epsilon}\right)
$$

This approach was suggested in 1990-91, firstly for the formal calculations in the stationary regime by Sone, then for regular regimes and finite times by Marra, Esposito and Lebowitz and finally by Bardos, Golse and Levermore (see [2]), inspired by the derivation of the diffusion approximation for Equation (15) by the method of moments. This calculation was, at the time, formal and it was justified only for very regular and small initial data in relation to the viscosity by Bardos and Ukai in 1991.

The final goal, which was the proof of convergence for any time and any "natural" initial data, was the object of various contributions and it was only completely achieved in 2004 by Golse and Saint-Raymond [13].

In [13], the authors use, in addition to the deduced estimates of energy and entropy, a control of the acoustic waves (given by P.-L. Lions and Masmoudi [14]), as well as a refined version of the averaging lemmas. They demonstrate that, for a family of solutions of the free transport equation, the properties of equi-integrability in the variables $v$ can be transposed into equi-integrability in the variables $(x, v)$ in a way that, with the Dunford-Pettis theorem, obtains strong convergence. This results from the following proposition.

Proposition 4.1. For all $1 \leq p \leq q \leq \infty$, every solution $f$ of the equation

$$
\partial_{t} f+v \cdot \nabla_{x} f=0 \quad \text { on } \mathbb{R} \times \mathbb{R}_{x}^{d} \times \mathbb{R}_{v}^{d}
$$

satisfies the estimate

$$
\|f(t)\|_{L_{x}^{q}\left(L_{v}^{p}\right)} \leq|t|^{-d\left(\frac{1}{p}-\frac{1}{q}\right)}|f(0)|_{L_{x}^{\infty}\left(L_{v}^{p}\right)} .
$$

\section{Averaging lemmas and the Wigner transform}

In Proposition 4.1, the reader will note an astonishing similarity with the properties of regularisation of the free Schrödinger equation (and its Strichartz inequalities) that are obtained by 
representing, for $t>0$, the solution of the free Schrödinger equation in $\mathbb{R}^{d}$

$$
i \partial_{t} \psi+\frac{1}{2} \Delta \psi=0
$$

by the formula

$$
\psi(x, t)=\frac{e^{-i \frac{d \pi}{4}}}{(2 \pi t)^{\frac{d}{2}}} \int_{\mathbb{R}^{d}} e^{-\frac{|x-y|^{2}}{2 i t}} \psi_{0}(y) d y,
$$

from which can be deduced, on the one hand, the regularising effect (for example, for every distribution with compact support, the function $\psi(x, t)$ is analytic) and on the other hand, the effect of dispersion:

$$
\|\psi(\cdot, t)\|_{L^{\infty}\left(\mathbb{R}^{d}\right)} \leq \frac{1}{(2 \pi t)^{\frac{d}{2}}} \int_{\mathbb{R}^{d}}\left|\psi_{0}(y)\right| d y .
$$

Taking into account the relation

$$
\frac{d}{d t}\|\psi(\cdot, t)\|_{L^{2}\left(\mathbb{R}^{d}\right)}=0,
$$

one can deduce the classical Strichartz estimates.

The relation between the dispersion effect for the transport equation and the Schrödinger equation was described in detail for the first time by Castella and Perthame [5]. It is naturally explained by considering the Wigner transform $w(x, v, t)$ [11], which allows the definition of a "quantum kinetic theory" in the "phase space" $\left(\mathbb{R}_{x}^{d} \times \mathbb{R}_{v}^{d}\right)$ by introducing the Wigner function:

$$
w(x, v, t)=\frac{1}{(2 \pi)^{d}} \int_{\mathbb{R}_{y}^{d}} \psi\left(x+\frac{y}{2}\right) \psi^{*}\left(x-\frac{y}{2}\right) e^{-i v \cdot y} d y,
$$

which transforms the complex-valued wave function into a real-valued function on the phase space, with the inconvenience that $w$ may be negative. It is not a probability distribution in the strict sense like $f$ in the classical kinetic equations. Apart from that, the Wigner function has all the good properties, with, for example, the moments in $v$ giving macroscopic densities like densities of "position"

$$
\int_{\mathbb{R}_{v}^{d}} w(x, v, t) d v=|\psi(x, t)|^{2}=\rho(x, t)
$$

and "current"

$$
\int_{\mathbb{R}_{v}^{d}} v w(x, v, t) d v=\mathfrak{I m}\left(\nabla \psi(x, t) \psi^{*}(x, t)\right)=J(x, t) .
$$

The free Schrödinger equation (30) transforms directly into the free transport equation

$$
i \partial_{t} \psi+\frac{1}{2} \Delta \psi=0 \quad \Rightarrow \quad \partial_{t} w(x, v, t)+v \cdot \nabla_{x} w(x, v, t)=0,
$$

from which we deduce again (32), noting that

$$
\begin{aligned}
|\psi(x, t)|^{2} & =\int_{\mathbb{R}_{v}^{d}} w(x, v, t) d v \\
& =\int_{\mathbb{R}_{v}^{d}} w_{0}(x-v t, v, t) d v .
\end{aligned}
$$

In the same way, in the papers by Perthame, Gasser and Markowich (see [17]), there is a systematisation from the kinetic point of view, in order to find the dispersion estimates, as well as a generalisation of that method for other PDEs.

More generally, with a real potential $V$ and Planck constant $\hbar$ (the limit $\hbar \rightarrow 0$ of which represents the "(semi) classical" limit), $H_{\hbar} \psi=-\frac{\hbar^{2}}{2} \Delta \psi+V \psi$ defines a Hamiltonian operator (unbounded but selfadjoint) on the space $L^{2}\left(\mathbb{R}^{d}\right)$. Then, the solution of the Schrödinger equation

$$
i \hbar \partial_{t} \psi_{\hbar}=-\frac{\hbar^{2}}{2} \Delta \psi_{\hbar}+V \psi_{\hbar}
$$

is given by $\psi_{0} \mapsto \psi_{\hbar}(t)=e^{-i \frac{t}{\hbar} H_{\hbar}} \psi_{0}$, with $e^{-i t H_{\hbar}}$ being a unitary group.

Now, the time evolution of the Wigner function is given by the Wigner equation consisting of the classical free transport operator and a pseudodifferential operator in $V$ (clearly nonlocal due to the Fourier transform in definition (33)):

$$
\partial_{t} w_{\hbar}(x, v, t)+v \cdot \nabla_{x} w_{\hbar}(x, v, t)-\left[\Theta\left(V_{\hbar}\right) w_{\hbar}\right](x, v, t)=0,
$$

where $\left.\Theta\left(V_{\hbar}\right) w_{\hbar}\right](x, v, t)$ is given by the integral in $\left(y, v^{\prime}\right)$ of the product of

$$
\frac{1}{(2 \pi)^{d}} \frac{V\left(x+\frac{\hbar y}{2}\right)-V\left(x-\frac{\hbar y}{2}\right)}{i \hbar} e^{-i v \cdot y}
$$

and

$$
e^{i \frac{v^{\prime} \cdot(x-y)}{\hbar}} w_{\hbar}\left(\frac{x+y}{2}, v^{\prime}, t\right) .
$$

For a sufficiently regular potential $V$, one has

$$
\left[\Theta\left(V_{\hbar}\right) w_{\hbar}\right](x, v, t)=\nabla_{x} V(x) \nabla_{v} w_{\hbar}(x, v, t)+O(\hbar) .
$$

So, we recover, at least formally, the Liouville equation, in the limit $\hbar \rightarrow 0$. It can be demonstrated that, under certain hypotheses, the "Wigner function" $w_{\hbar}(x, v, t)$ converges to a non-negative measure $w_{0}(x, v, t)$, called "the Wigner measure" by P.-L. Lions and Paul [15], and, for this $w_{0}(x, v, t) \geq 0$, we recover, at least formally, classical kinetic theory, i.e., the Liouville equation

$$
\partial_{t} w_{0}(x, v, t)+v \cdot \nabla_{x} w_{0}(x, v, t)-\nabla_{x} V \cdot \nabla_{v} w_{0}(x, v, t)=0 .
$$

The above derivations can be rigorously justified: the general theory for the linear case is elaborated in [11] and the special nonlinear case for the limit of the "Schrödinger-Poisson" system toward "Vlasov-Poisson" is given in [15] and [25].

We would like to close this brief presentation of "quantum kinetics" with the following comments.

(1) The Wigner function is a reformulation of the "density matrix" $K_{\hbar}(x, y, t)$, defined for a "pure state" $\psi$ like $K_{\hbar}(x, y, t)=\psi_{\hbar}(x, t) \otimes \psi_{\hbar}^{*}(y, t)$. This density matrix, which is the kernel of an integral operator in $L^{2}\left(\mathbb{R}^{d}\right)$ named the "density operator" $\hat{K}$, is a key object of statistical quantum mechanics. In the general case of a "mixed state", where the system is found with a probability $\lambda_{j}$ in the state $\psi_{j}(x, t)$, with $\lambda_{j} \geq 0$ and $\sum_{j=1}^{\infty} \lambda_{j}=1$, one has

$$
K_{\hbar}(x, y, t)=\sum_{j=1}^{\infty} \lambda_{j}\left(\psi_{j}\right)_{\hbar}(x, t) \otimes\left(\psi_{j}\right)_{\hbar}^{*}(y, t) .
$$

While the operator $\hat{K}_{\hbar}$ is a solution of the Heisenberg-von Neumann equation

$$
i \hbar \frac{d}{d t} \hat{K}_{\hbar}+\left[H_{\hbar}, \hat{K}_{\hbar}\right]=0
$$

the relation between the density matrix $K_{\hbar}(x, y, t)$ and the Wigner function $w_{\hbar}(x, v, t)$ is given by

$$
K_{\hbar}(x, y, t)=\int_{\mathbb{R}_{v}^{d}} e^{i \frac{v \cdot(x-y)}{\hbar}} w_{\hbar}\left(\frac{x+y}{2}, v, t\right) d v
$$


and

$$
w_{\hbar}(x, v, t)=\frac{1}{(2 \pi)^{d}} \int_{\mathbb{R}_{y}^{d}} e^{-i v \cdot y} K_{\hbar}\left(x+\frac{\hbar y}{2}, x-\frac{\hbar y}{2}, t\right) d y .
$$

With $\hbar \rightarrow 0$, the formal calculation above yields a correspondence between the evolution of $\hat{K}_{\hbar}$ according to the Heisenberg-von Neumann equation (38) and the evolution of $w=\lim _{\hbar \rightarrow 0} w_{\hbar}$ according to the (kinetic) Liouville equation:

$$
\partial_{t} w+\{H, w\}=0 \text {. }
$$

We see how, within the "(semi)classical limit", the commutator of the operators becomes the Poisson bracket (2) of the functions.

(2) Under the hypotheses of convenient regularity of $w$, we find, as part of Weyl calculus, the theorem obtained by Egorov in 1970 [9], using the integral Fourier operators introduced by Hörmander. This theorem precisely states that, in this setting, the operator $e^{-i \frac{t}{\hbar} \hat{H}_{\hbar}} K_{\hbar}(0) e^{i \frac{t}{\hbar} \hat{H}_{\hbar}}$ is, up to a computable rest, described by Formula (39), with $w$ being the solution of Equation (40).

The main lines of the computation above remain formally valid in the nonlinear case, where the potential $V$ depends on the solution as in the Heisenberg-von Neumann-Poisson case:

$$
\begin{aligned}
& i \frac{d}{d t} \hat{K}_{\hbar}+\left[-\frac{\hbar^{2}}{2}+V_{\hbar}, \hat{K}_{\hbar}\right]=0, \\
& V_{\hbar}(x)=\frac{1}{4 \pi} \int_{\mathbb{R}_{x}^{3}} \frac{1}{|x-y|} K_{h}(y, y) d y,
\end{aligned}
$$

that is,

$$
-\Delta V_{\hbar}=K_{h}(x, x)=\operatorname{Trace}\left(\hat{\mathrm{K}}_{\hbar}\right)
$$

We thus obtain, in the limit $\hbar \rightarrow 0$, the Vlasov-Poisson equation

$$
\begin{aligned}
& \partial_{t} w+w \cdot \nabla_{x} w-\nabla_{x} V \cdot \nabla_{v} w=0, \\
& \rho(x, v, t)=\int_{\mathbb{R}_{v}^{3}} w(x, v, t) d v, \\
& -\Delta V(x, t)=\rho(x, t) .
\end{aligned}
$$

Because of the problem's nonlinearity, the justification, in the existing proofs of taking the limit $\hbar \rightarrow 0$, requires that the Wigner transform of the initial datum $K_{h}(0)$ is uniformly bounded in $L^{2}$. In one space dimension, this was realised for weak (non-unique) solutions of Vlasov-Poisson with measure valued initial datum by Zheng, Zhang and Mauser [25]. But, in higher dimensions, as stated in the papers by P. L. LionsPaul and Markowich-Mauser, it seems unavoidable to consider mixed states (37), with a very restrictive condition on the $\lambda_{j}$, which must depend on the Planck constant $\hbar$ in a way that

$$
\sum_{j=1}^{\infty}\left(\lambda_{j}^{\hbar}\right)^{2} \leq C \hbar^{3} .
$$

Interestingly, we see that this idea already appears in 1946 in a notice by Yvon [24]. Actually, we find Jacques Yvon's presence throughout the whole history of this subject!

Taking into account the different fields of application, many mathematical variants of the averaging lemmas have appeared; for example, in [12], the following theorem underlines the role of a transversal hypothesis.
Theorem 5.1. Let $\mu$ be a bounded positive measure on $\mathbb{R}_{v}^{d}$ such that

$$
\sup _{v \in S^{d-1}} \mu\left(\left\{v \in \mathbb{R}_{v}^{d} /|v \cdot e| \leq \epsilon\right\}\right) \leq C \epsilon, \quad \forall \epsilon>0 .
$$

Then, with $u$ a solution of the equation $u+v \cdot \nabla_{x} u=f$, the map $f \mapsto \int u(x, v) d \mu(v)$ is continuous from $L^{2}(d x \otimes d \mu(v))$ to $H^{\frac{1}{2}}\left(\mathbb{R}_{x}^{d}\right)$.

This result has been generalised by Gérard and Golse [10] for averages with respect to $y$ of solutions to pseudodifferential equations. In order to handle problems of VlasovMaxwell type, DiPerna and P. L. Lions [7] have, in the theory of kinetic equations, included averaging lemmas with differential operators in the variable $v$, and this point of view has been systematically generalised in the paper by Tadmor and Tao [20].

\section{Conclusions: kinetic equations and statistical limits}

The contributions mentioned above on the subject of averaging lemmas solely concerned the relations between kinetic and macroscopic equations. But, of course, their history also involves relations between the dynamics of $N$ particles (molecules, atoms, ions and electrons) with $N$ large or tending to infinity. One then enters into the realm of (classical or quantum) statistical mechanics, which could easily provide subjects for a lot of further articles.

We should mention that, in an intuitive and formal way, those relations could already be found in the spirit of Maxwell, Boltzmann and Lorentz: one of the most important tools was the BBGKY hierarchy, as introduced in this context by Yvon in 1935 [24], and the first rigorous works are due to Grad and Lanford.

It should also be noted that there are important similarities between the derivations of kinetic models from classical statistical mechanics and from quantum statistical mechanics. This can be explained by using the Wigner transformation. It is in this same context that the Vlasov equation mentioned above appears naturally.

We observe that Vlasov presented his equation in 1938, 66 years after the Boltzmann equations. This is explained by the fact that the Vlasov equation applies to modern domains of physics, while the Boltzmann equation was related to 19 th century physics. However, because of its really nonlinear character, it contains the theory's essential difficulties.

After the pioneering works of Neunzert and Spohn (see [19]), the subject developed within the same community, also using the tools referred to above (for example, the version of the averaging lemmas given in [7]). Moreover, Vlasov's approach has morphed into new shapes motivated by contemporary physics, in particular in the modelling of plasmas, for instance in the context of nuclear fusion (the international project ITER, between Japan and Cadarache). A relativistic version of Vlasov's equation, where the force $F$ is the Lorentz force containing the magnetic field, and where the speed $v$ is bounded by the speed of light $c$ and given by $v(\xi)$ with $\xi \in \mathbb{R}^{d}$, yields the nonlinear "Vlasov-Maxwell" system. Furthermore, there are "semi-non-relativistic" approaches of order $1 / c$ or $O\left(1 / c^{2}\right)$ (for a concise presentation of this hierarchy of non- 
linear kinetic equations, see, for example, [4]). We notice that, for this kind of kinetic equation, a lot of problems are still unsolved in mathematical and numerical analyses.

Finally, as the Liouville equation (1) generates a Hamiltonian flow that preserves the measure on the phase space, there have been recent works (Brenier et al.) on the relations between Vlasov-Monge-Ampère equations and the optimal transport equation (see [21]).

With these observations in mind, it is clear that, since the decade of 2000, works on this subject multiplied with, amongst others, the work by Mouhot and Villani in 2011 on Landau damping [16], which contributed to a second Fields Medal for the French kinetic community. The European network HYKE (HYperbolic and Kinetic Equations, 2002-2005), with its 350 researchers in 16 European countries and the USA, amongst them nearly all the mathematicians cited in this article (and some young fellow researchers that left us too soon, like N. BenAbdallah and F. Poupaud), acted as an important catalyst in this evolution.

\section{Acknowledgements}

The authors would like to thank in particular Thomas Alazard for his precious advise and help in the redaction, as well as the unknown referees and M. Farge, F. Golse, J. C. Saut and R. Sentis for constructive criticism. Also we thank J.-B. Bru and M. Gellrich Pedra for their great job with the English translation. Financial support of the FWF ("Austrian Science Foundation") is acknowledged under project No. F65 (SFB "Taming Complexity in PDEs") and W1245 (DK "Nonlinear PDEs").

\section{Bibliography}

[1] C. Bardos, R. E. Caflisch and B. Nicolaenko, The Milne and Kramers problems for the Boltzmann equation of a hard sphere gas. Comm. Pure Appl. Math. 39 (3) (1986), 323-352.

[2] C. Bardos, F. Golse and D. Levermore, Fluid Dynamic Limits of Kinetic Equations I: Formal Derivations. J. Stat. Phys. 63 (1991), 323-344.

[3] B. J. Bayly, C. D. Levermore and T. Passot, Density variations in weakly compressible fluid flow. Phys. Fluids A 4 (1992), 945-954.

[4] N. Besse, N. J. Mauser and E. Sonnendrucker, Numerical approximation of self-consistent Vlasov models for lowfrequency electromagnetic phenomena. Intl. J. of Appl. Math. and Comput. Sciences 17 (3) (2007), 1-20.

[5] F. Castella and B. Perthame, Estimations de Strichartz pour les equations de transport cinétique. C. R. Acad. Sci. Paris Sr. I Math. 322 (6) (1996), 535-540.

[6] R. DiPerna and P. L. Lions, On the Cauchy problem for Boltzmann equations: global existence and weak stability. Ann. of Math. (2) 130 (1989), 321-366.

[7] R. DiPerna and P. L. Lions, Global weak solutions of VlasovMaxwell systems. Comm. Pure Appl. Math. 42 (6) (1989), 729-757.

[8] E. Chiodaroli, C. De Lellis and O. Kreml, Global ill-posedness of the isentropic system of gas dynamics. Comm. Pure Appl. Math. 68 (7) (2015), 1157-1190.

[9] J. V. Egorov, On the local solvability of pseudo-differential equations. Actes ICM Nice 1970, Tome 2, pp. 717722. Gauthier-Villars, Paris (1971).
[10] P. Gérard and F. Golse, Averaging regularity results for PDEs under transversality assumptions. Comm. Pure Appl. Math. 45 (1) (1992), 126.

[11] P. Gérard, P. A. Markowich, N. J. Mauser and F. Poupaud, Homogenization limits and Wigner transforms. Comm. Pure Appl. Math. 50 (1997), 321-377.

[12] F. Golse, P. L. Lions, B. Perthame and R. Sentis, Regularity of the moments of the solution of a transport equation. J. Funct. Anal. 76 (1) (1988), 110-125.

[13] F. Golse and L. Saint-Raymond, The Navier-Stokes limit of the Boltzmann equation for bounded collision kernels. Invent. Math. 155 (1) (2004), 81-161.

[14] P.-L. Lions and N. Masmoudi, From the Boltzmann equations to the equations of incompressible fluid mechanics. I and II. Arch. Ration. Mech. Anal. 158 (2001), 173-193 and 195-211.

[15] P.-L. Lions and T. Paul, Sur les mesures de Wigner. Rev. Mat. Iberoamericana 9 (3) (1993), 553-618.

[16] C. Mouhot and C. Villani, On Landau damping. Acta Math. 207 (1) (2011), 29-201.

[17] B. Perthame, Mathematical tools for kinetic equations. Bull. Amer. Math. Soc. (N.S.) 41 (2) (2004), 205-244.

[18] R. Sentis, The principal eigenvalue of a transport operator an asymptotic expansion. System Modeling and Optimization (New York, 1981), 393-400, Lecture Notes in Control and Inform. Sci., 38, Springer, Berlin, (1982).

[19] H. Spohn, The derivation of kinetic equations from Hamiltonian dynamics - the example of the Lorentz gas. In: Mathematical Problems in the Kinetic Theory of Gases (Oberwolfach 1979), eds. Pack and Neunzert, Peter Lang Verlag (1980).

[20] E. Tadmor and T. Tao, Velocity averaging, kinetic formulations, and regularizing effects in quasi-linear PDEs. Comm. Pure Appl. Math. 60 (10) (2007), 1488-1521.

[21] C. Villani, Optimal Transport. Old and New. Springer, Berlin (2009).

[22] S. Weinberg and E. Wigner. The Physical Theory of Neutron Chain Reactors. The University of Chicago Press (1958).

[23] J. Yvon, La théorie statistique des fluides et l'équation d'état. Act. Scient. ind. 203, 50 pages, Hermann Paris (1935).

[24] J. Yvon, Sur les rapports entre la théorie des mélanges et la statistique classique. C. R. Acad. Sci. Paris (1946), 347-349. Il s'agit probablement de la publication dont le manuscript avait été écrit en déportation en Mars 1945.

[25] P. Zhang, Y. Zheng and N. J. Mauser, The limit from the Schrödinger-Poisson to the Vlasov-Poisson equations with general data in one dimension. Comm. Pure Appl. Math. 55 (2002), 582-632.

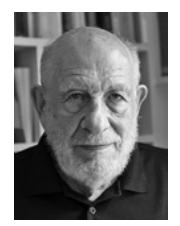

Claude Bardos [claude.bardos@gmail.com] is a professor emeritus of mathematics and a specialist in PDEs in physics and fuid dynamics. www.ljll.math.upmc.fr/ bardos/

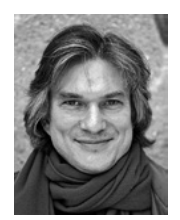

Norbert J. Mauser [mauser@courant.nyu. edu] is a professor of mathematics and a specialist in PDEs and quantum physics. www. wpi.ac.at/director

This is the English translation of the French article "Équations cinétiques: une histoire française", published in La Gazette des Mathématiciens, Société Mathématique de France (No. 155, pp. 25-37, 2018). The EMS Newsletter thanks La Gazette des Mathématiciens for permission to republish this article. 